\title{
CHIMERAS ON A SOCIAL-TYPE NETWORK
}

\author{
ARKADY PIKOVSKY ${ }^{1,2, *}$
}

\begin{abstract}
We consider a social-type network of coupled phase oscillators. Such a network consists of an active core of mutually interacting elements, and of a flock of passive units, which follow the driving from the active elements, but otherwise are not interacting. We consider a ring geometry with a long-range coupling, where active oscillators form a fluctuating chimera pattern. We show that the passive elements are strongly correlated. This is explained by negative transversal Lyapunov exponents.
\end{abstract}

Mathematics Subject Classification. 37N99, 37M05.

Received September 18, 2020. Accepted February 3, 2021.

\section{INTRODUCTION}

Since their discovery about 20 years ago by Kuramoto and Battogtokh [7], chimera patterns attracted large interest in studies of complex systems. Chimera is an example of a symmetry breaking in a homogeneous system of coupled oscillators: together with a homogeneous fully synchronous state there exist non-homogeneous states where some oscillators are synchronized and some not. In spatially extended systems chimera appears as a localized pattern of asynchrony [1-3, 8-13, 18, 21]. In globally coupled populations chimeras are also possible: they emerge not as spatial patterns, rather a group of asynchronous oscillators "detaches" from the synchronous cluster $[6,16,17,22,23]$.

The basic model of Kuramoto and Battogtokh is a one-dimensional ring of phase oscillators with non-local coupling. Each oscillator is coupled to all others in a symmetric bidirectional way; the strength of coupling depends on the distance on the ring. There are two typical setups for this distance dependence: exponential as in [7] (or its modification taking into account spatial periodicity [18]), or cos-shape coupling [1]. In both cases, chimera lives on a symmetric weighted bidirectional network. This paper aims to generalize the basic setting of the Kuramoto and Battogtokh to a social-type network (STN). Such a network, introduced in [14], deserves a detailed description. It is a weighted directional network with two types of nodes: (i) active nodes that force other nodes and potentially are also forced by them (i.e., active nodes have outgoing links); (ii) passive nodes that are driven by active nodes but do not influence them (i.e., passive nodes have only in-going links). We illustrate this in Figure 1. The name "social-type" is picked because a separation into active and passive nodes is similar to the separation of social networks into "influencers" and "followers". The latter participants get input from the former ones, but not vice versa. In physics, there are several prominent models of such type.

Keywords and phrases: Network, Chimera, correlations, Lyapunov exponent.

1 Department of Physics and Astronomy, University of Potsdam, 14476 Potsdam-Golm, Germany.

2 Department of Control Theory, Lobachevsky University of Nizhny Novgorod, Gagarin Avenue 23, 603950 Nizhny Novgorod, Russia.

* Corresponding author: pikovsky@uni-potsdam.de 


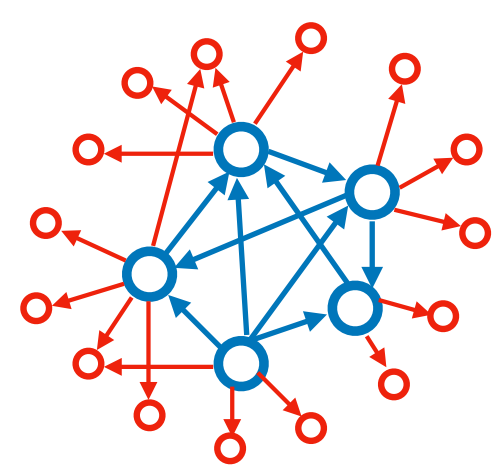

FIGURE 1. Illustration of a social-type network. Central blue/big units are active, they interact with each other, but do not get inputs from passive units (peripherial red/small ones). The passive units are driven by the active ones, and do not interact with each other.

In a restricted many-body problem in celestial mechanics, one considers several heavy bodies that interact and move according to the gravitational forces they produce. Additionally, light bodies move in the gravitational field created by the heavy ones but do not produce gravitational forces themselves (in fact, these forces are neglected in this setup). Another situation is modeling of a two-dimensional turbulence by a motion of point vortices [4]. The vortices move as interacting fluid particles, while other particles, like passive tracers, follow the velocity field created by the vortices but do not contribute to it.

Below we construct the STN by taking a symmetric Kuramoto-Battogtokh network, and equipping it with additional passive oscillators. We will mainly consider a situation where the number of passive units is much larger that the number of active ones. The model will be introduced in Section 2. In Section 3 we will illustrate the dynamics of passive units, and in Section 4 will perform its statistical evaluation.

\section{BASIC MODEL}

We consider a network consisting of $N$ active phase oscillators $\varphi_{n}(t)$ and $M$ passive phase oscillators $\vartheta_{m}(t)$. Both are uniformly distributed in space on a ring $[0,1)$, the coordinates of active units are $x_{n}=(n-1) / N$, $n=1, \ldots, N$; the coordinates of passive units are $y_{m}=(m-1) / M, m=1, \ldots, M$. All oscillators have identical frequencies (which we set to zero chosing the appropriate rotating reference frame), and are nonlocally coupled:

$$
\begin{aligned}
& \dot{\varphi}_{n}(t)=\frac{1}{N} \sum_{k=1}^{N} G\left(x_{k}-x_{n}\right) \sin \left(\varphi_{k}(t)-\varphi_{n}(t)-\alpha\right), \quad n=1, \ldots, N \\
& \dot{\vartheta}_{m}(t)=\frac{1}{N} \sum_{k=1}^{N} G\left(x_{k}-y_{m}\right) \sin \left(\varphi_{k}(t)-\vartheta_{m}(t)-\alpha\right), \quad m=1, \ldots, M
\end{aligned}
$$

One can see that this coupling implements an STN: while active oscillators are mutually coupled, passive ones just follow the force from the active ones.

In previous literature, several coupling kernels $G(\cdot)$ has been explored. Kuramoto and Battogtokh [7] used an exponential kernel, Abrams and Strogatz [1] used a cos-shaped one. We will follow the latter option, and set $G$ as

$$
G(x)=1+A \cos (2 \pi x) .
$$

Parameters $A=3 / 4$ and $\alpha=\pi / 2-0.05$ are fixed throughout the paper. 
Nontrivial properties in the social-type network (2.1) and (2.2) can be expected if the number of active oscillators $N$ is not too large. Indeed, in the thermodynamic limit $N \rightarrow \infty$ the field created by active oscillators is stationary (in a certain rotating reference frame), and the dynamics of passive oscillators in this field is trivial. In contradistinction, for relatively small $N$ there are significant finite-size fluctuations, which, as we will see, lead to nontrivial effects. On the other hand, it is known that chimera in a very small population is a transient state [20]. Below in this paper we choose $N=32$; for the parameters chosen chimera in equations (2.1) and (2.3) is strongly fluctuating and has a long life time (we never observed any transition from chimera to synchrony at times up to $10^{7}$ ).

Next, we discuss the role of the number of passive units $M$. This parameter has no any influence on the dynamics, because the passive oscillators neither influence the active one nor influence each other. Thus one could take $M=1$ if one-particle statistical properties are of interest (like the transversal Lyapunov exponent addressed below in Sect. 4.2), or $M=2$ if a two-particle statistics is of interest (like in calculation of the crosscorrelations in Sect. 4.1). Moreover, the positions of passive units can be arbitrary. However, for better visualization (like for a visualization of a fluid flow one uses many passive tracers from smoke or dye) and for gathering enough statistics already in one or several runs, it is convenient to take as many passive units as it is possible in a numerical implementation. Therefore, below we mostly take $M=8192 \gg N=32$.

\section{VisualizATION OF CHIMERA}

In Figure 2a we illustrate the chimera regime in the set of active units $\varphi_{k}$. We show the distance between the states of neighboring active oscillators $D_{k}=\left|\sin \left(\frac{\varphi_{k+1}-\varphi_{k}}{2}\right)\right|$. This quantity is close to zero if the phases $\varphi_{k}$ and $\varphi_{k+1}$ are nearly equal, and is 1 if the phase difference is $\pi$. In Figure 2a the black region corresponds to a coherent domain of chimera (all the phases here are nearly equal), and the rest with red/yellow colors is the disordered state.

Next we illustrate what happens to passive oscillators in the regime depicted in Figure 2a. In Figure $2 \mathrm{~b}$ we show the corresponding space-time plot, and in Figure 3 we show a snapshot of the states of active and passive oscillators. It has following features:

1. First we mention that the passive elements which have exactly the same positions as the active ones, attain the same state. This is due to the fact that although initial conditions are different, these pairs are driven by exactly the same field, and the conditional Lyapunov exponents are negative (see a detailed discussion of Lyapunov exponents below in Sect. 4.2), so that active and passive oscillators synchronize.

2. The active oscillators show typical for chimera domains where the phases are nearly equal (here $0.18 \lesssim$ $x \lesssim 0.4$ ), and another one, where neighboring elements do not have close phases. In contradistinction, close in space passive oscillators typically have also close values of the phases. This is due to the fact, that they receive nearly the same driving (if their positions are closed), and their dynamics is stable (as it follows from the negative values of the conditional Lyapunov exponent); as a result they nearly synchronize. Visually this appears in a snapshot as a continuous profile of passive phases values. Of course, this profile cannot be exactly continuous because of phase slips, which are also clearly visible in Figure 3 (e.g., at $x \approx 0.21$ and at $x \approx 0.24$ ); in the space-time plot Figure $2 \mathrm{~b}$ these slips correspond to narrow lines on the black background. Such a phase slip disappears due to finite spacing between passive elements and the stabilizing role of the negative Lyapunov exponent (see Appendix for a detailed discussion). Thus, passive oscillators possess certain degree of regularity also in the domain where active oscillators are disordered. This degree, however, decreases with the distance and one does not observe this regularity for active oscillators - they are too distant and receive quite different driving.

\section{Statistical Properties}

\subsection{Cross-correlations}

To characterize the level of regularity of passive units, we calculate the cross-correlation between the phases. Here, as has been shown in reference [14], it is important to use a proper observable. Indeed, because the rotations 
(a)

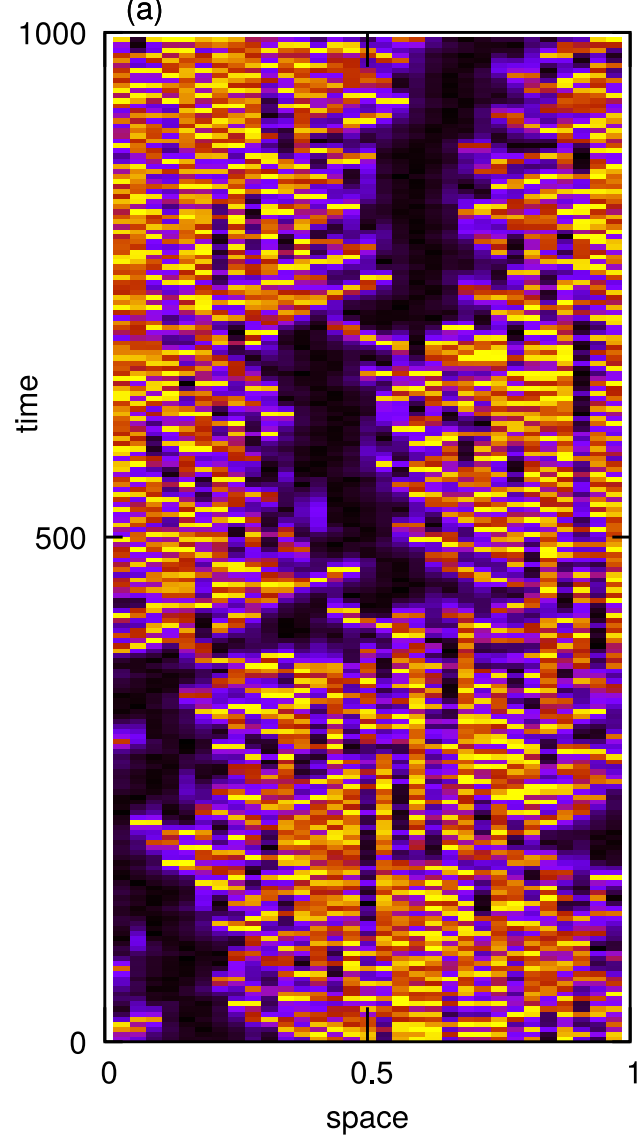

(b)

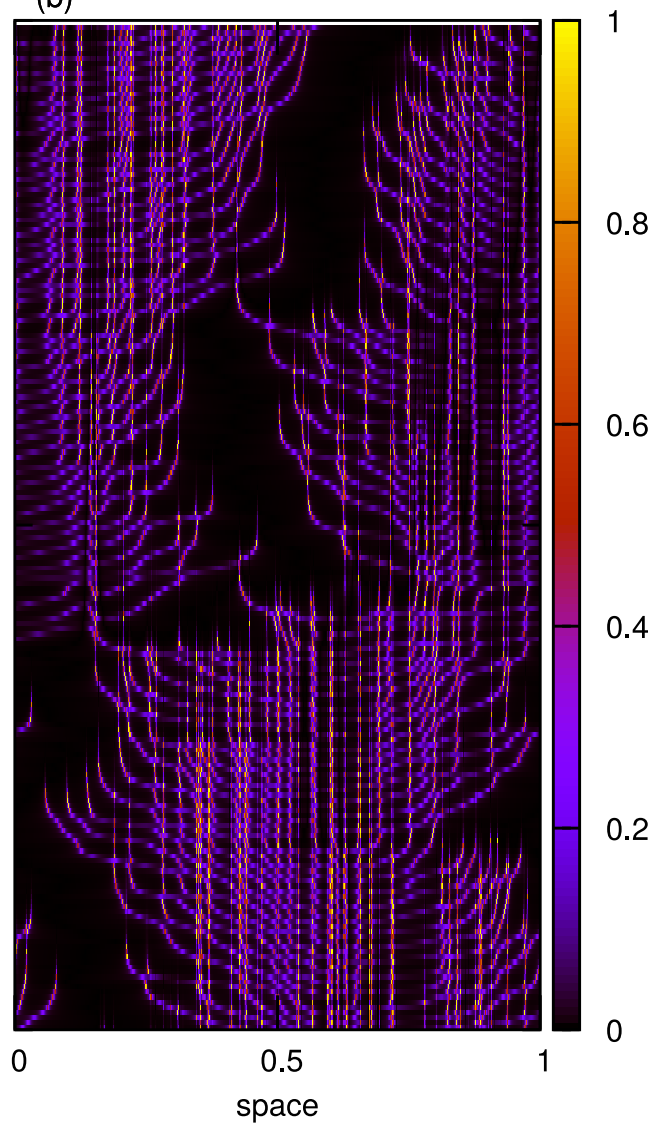

Figure 2. Panel (a): Chimera in a set of 32 active units (2.1). Color coding shows the distances between neighboring units $D_{k}$, as function of time. Black region corresponds to a synchronized domain, yellow-red irregular pattern to the desynchronized one. The position of the synchronized domain experiences a random walk, so that the dynamics on the long time scale is ergodic - each oscillators participates in synchronous and asynchronous motions. Panel (b): Distance between the $M=1024$ passive neighbors $D_{m}=\left|\sin \left(\frac{\vartheta_{m+1}-\vartheta_{m}}{2}\right)\right|$ for the same run as in panel (a). One can see much larger domains of black color indicating for close values of the passive phases, interrupted by narrow red lines corresponding to slips.

of passive phases are not free, their distribution is not uniform - this can be clearly seen in Figure 3, where the phases in the disordered domain are concentrated around the value $\vartheta \approx 2.5$. In reference [14], where the Kuramoto model on a STN was treated, the transformation from the inhomogeneous phase $\vartheta$ to a homogeneous observable $\theta$ was performed using the local instantaneous complex order parameter $z=\left\langle e^{i \vartheta}\right\rangle_{l o c}$ by virtue of the Möbius transform

$$
\exp [i \theta]=\frac{\exp [i \vartheta]-z}{1-z^{*} \exp [i \vartheta]}
$$

In the chimera setup of this paper, we cannot properly define a local complex order parameter due to strong finite-size fluctuations. Instead, we use transformation (4.1) with the global order parameter of active 


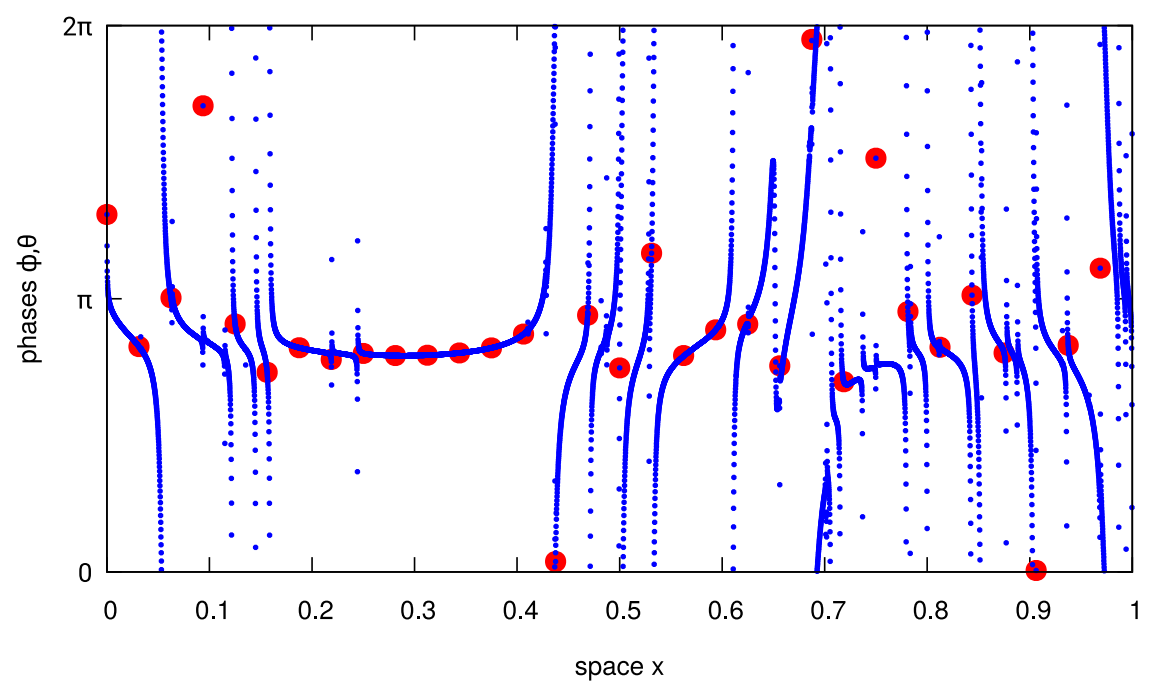

Figure 3. A snapshot of an STN with $N=32$ active units ( $\varphi_{k}$, large red filled circles) and $M=8192$ passive units $\left(\vartheta_{m}\right.$, blue dots $)$.

oscillators

$$
z=\frac{1}{N} \sum_{n} e^{i \varphi_{n}}
$$

After the transformation (4.1) is performed, the cross-correlation between passive oscillators is calculated according to

$$
c\left(\frac{m}{M}\right)=\left|\left\langle e^{i\left(\theta_{k}-\theta_{k+m}\right)}\right\rangle\right|
$$

where the averaging is performed over all the pairs of passive phases and over a long time interval. The latter has been chosen long enough that every oscillator was both in regular and irregular domains. The correlation function (4.2) is shown in Figure 4, for $N=32$ and $M=8192$. One can see that the correlation function tends to one as $\Delta y=\frac{m}{M}$ tends to zero, what corresponds to the mentioned above continuity of the phase profiles. At large $\Delta y$ the correlations are low; this is the advantage of using the "cleansed" observable $\theta$ instead of the original phase $\vartheta$, for the latter the cross-correlations do not drop below 0.4 .

\subsection{Lyapunov exponents}

In the context of STNs, there is a twofold application of the Lyapunov exponents (LEs). Usual LEs can be defined for a set of active particles, some of them are positive what corresponds to turbulent dynamics depicted in Figure 2. For passive oscillators, the LEs have a meaning of transversal Lyapunov exponents. Indeed, because passive units do not act on other oscillators, the system (2.1) and (2.2) is a skew one, and linearization of equation (2.2) for passive oscillators leads to a set of independent one-dimensional equations for perturbations

$$
\dot{\delta} \vartheta_{m}=-\delta \vartheta_{m} \frac{1}{N} \sum_{k=1}^{N} G\left(x_{k}-y_{m}\right) \cos \left(\varphi_{k}-\vartheta_{m}-\alpha\right),
$$




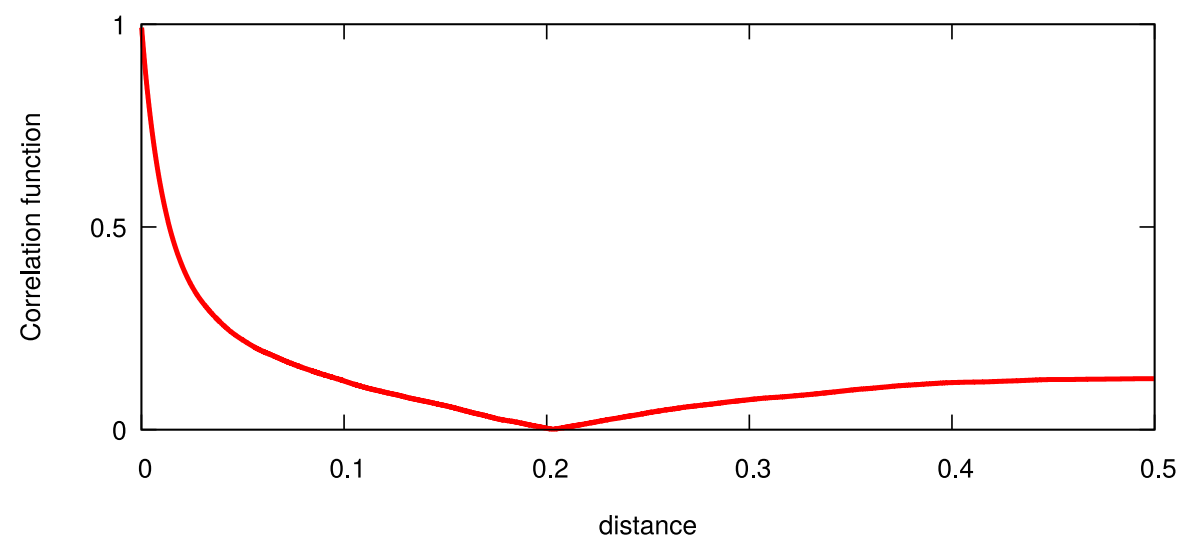

Figure 4. Cross-correlations in a chimera regime with $N=32$ active and $M=8192$ passive units, calculated according to (4.2).

from which the transversal LEs (they depend on the position $y_{m}$ ), can be expressed as

$$
\lambda_{t}(y)=-\left\langle\frac{1}{N} \sum_{k=1}^{N} G\left(x_{k}-y_{m}\right) \cos \left(\varphi_{k}-\vartheta_{m}-\alpha\right)\right\rangle .
$$

Calculated in this way transversal LEs are shown in Figure 5. They are all negative, with the minimum at the central position between the active units.

The interpretation of the transversal LEs is as follows. If there are two passive units at exactly the same position on the ring but with different initial conditions, then they will eventually approach each other and synchronize. Quantity $\lambda_{t}$ gives the average rate of this exponential approach. In particular, if a passive unit is at the same position as an active one, they will synchronize with the average rate $\lambda_{t}(0)$. The result of this synchronization has been already discussed in Section 3.

Negative transversal LEs explain also correlations of neighboring passive units (Fig. 4). Indeed, neighboring sites (distance $\Delta y$ ) experience different forcing fields, therefore they cannot synchronize completely. Instead, one can write a model linear equation for the difference of states of passive units

$$
\Delta \vartheta \approx-\left|\lambda_{t}\right| \Delta \vartheta+\Delta h
$$

where

$$
\Delta h \approx \Delta y \frac{1}{N} \sum_{k=1}^{N} \frac{\partial G\left(x_{k}-y\right)}{\partial y} \sin \left(\varphi_{k}-\vartheta-\alpha\right)
$$

is the difference in the forcing. One can roughly estimate $\Delta \vartheta \approx \Delta h /\left|\lambda_{t}\right|$, i.e. neighboring passive units nearly synchronize for small $\Delta y$. This picture is however, not exact, as the discussion in Section 4.3 shows.

\subsection{Intermittency of satellites}

Here we focus on passive units that are extremely close to the active ones. We call them "satellites", and the corresponding active unit is their "host". In the Kuramoto model, such satellites are perfectly synchronized to the host [14] (similar to the restricted many-body problem in gravitational dynamics, where light particles in a vicinity of a heavy body do not leave this vicinity). In the present chimera setup, we however observe a different 


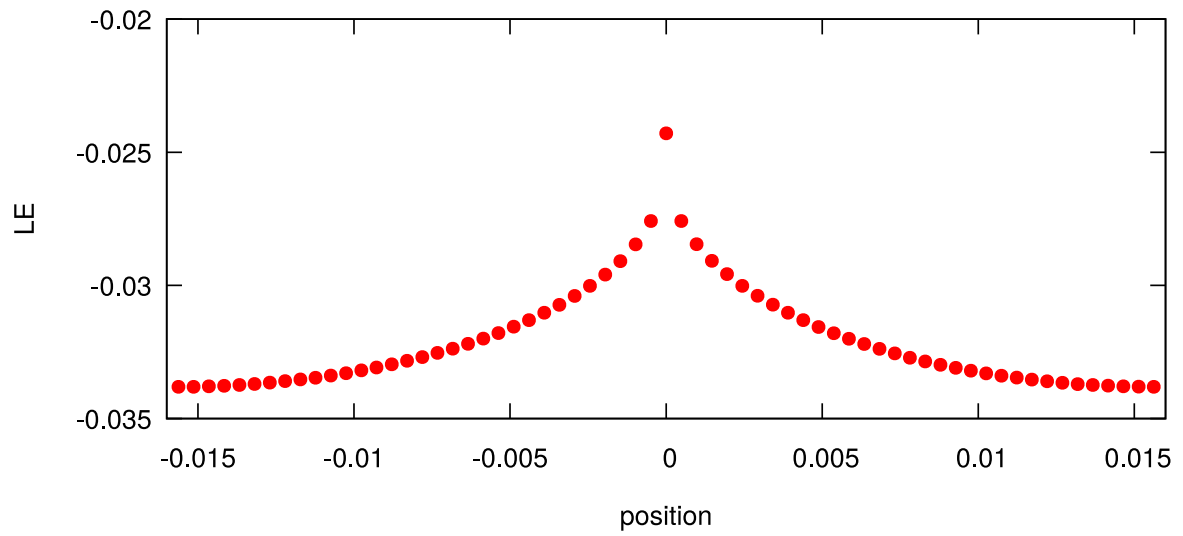

Figure 5. Transversal LEs vs position on the ring, for $N=32$. Due to periodicity with $1 / N$, only the interval $-1 / 2 N<y<1 / 2 N$ around an active unit at $y=0$ is shown.

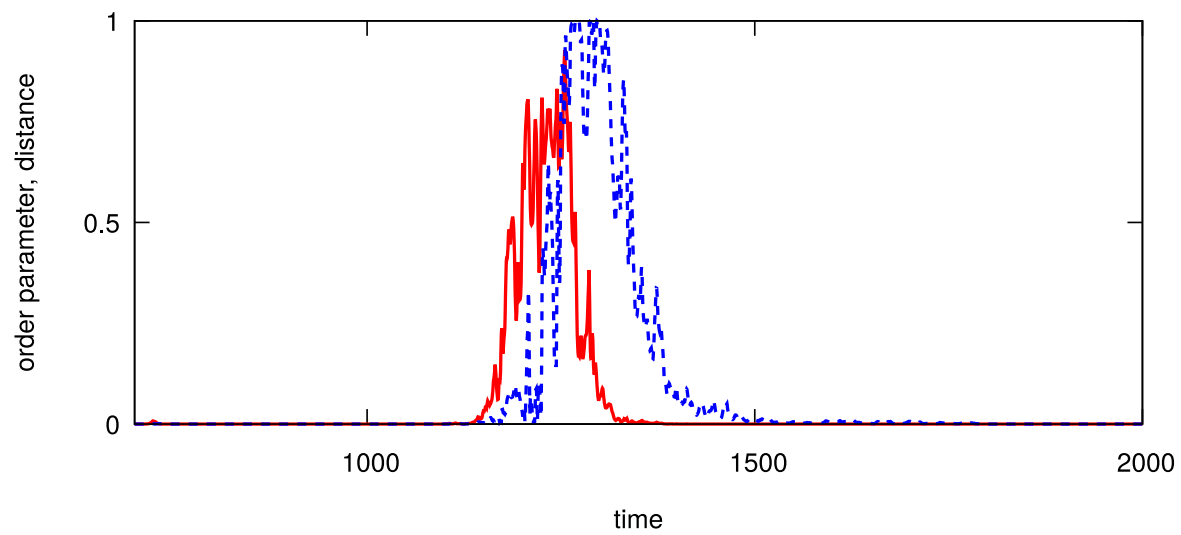

Figure 6. Illustration of intermittency in the satellites' dynamics. Red line: $1-\left|z_{s}\right|$; blue dashed line: distance $D$. Outside of the burst $1-\left|z_{s}\right| \approx D \approx 0$. The burst has three stages. (i) first $\left|z_{s}\right|$ decreases from one, but $D$ remains small; here the satellites are spread around the host. (ii) Both $1-\left|z_{s}\right|$ and $D$ are large, satellites are spread away from host. (iii) $\left|z_{s}\right| \approx 1$ but $D$ is large; satellites form a small cluster away from the host.

behavior. An inspection of Figure 3 shows that indeed in many cases the satellites are close to the hosts (these cases a represented by blue "lines" passing through red dots). However, there are at least three hosts which are detached from the satellites (these are isolated red dots at $x \approx 0.075,0.74,0.97$ ). Remarkably, the satellites near $x \approx 0.075,0.74$ lie close to the value $\vartheta \approx 2.5$ where the probability to find the phases at this moment of time is maximal; the satellites near $x \approx 0.97)$ lie close to the value $\vartheta \approx 0, i$. $e$. far away from the positions of the majority of phases.

Such a behavior is not covered by the simple relation (4.5). The reason is in the fluctuations of the transversal LE, not taken into account in relation (4.5). Such fluctuations may generally lead to so-called modulational intermittency [15], and this happens also here. On average the transversal LE is negative, but the values of quantity (4.4) averaged over a finite time interval may be positive. In this case an equation of type (4.5) results in an amplification of distances, and the satellites detach from the host. It may take a long transient time until they attach again. This process is indeed intermittent, as Figure 6 illustrates. In this figure we take $L=32$ passive satellites $\vartheta_{k}$ of an active host, which are spread in the vicinity of size $-10^{-5} \leq \Delta y \leq 10^{-5}$. To 
characterize these satellites, we calculate their complex order parameter (using cleansed phases $\theta$ )

$$
z_{s}=\frac{1}{L} \sum_{k=1}^{L} e^{i \theta_{k}}
$$

and depict in Figure 6, as functions of time, the absolute value $\left|z_{s}\right|$ and the distance from the host $\varphi$ measured as $D=\left|\sin \frac{\arg \left(z_{s}\right)-\varphi}{2}\right|$. Most of the time $\left|z_{s}\right| \approx 1$ and $D \approx 0$, what means that all the satellites are in a small neighborhood of the host. However, there is a burst where the satellites spread $\left(\left|z_{s}\right|\right.$ is as small as 0.2$)$ and detach from the host. At the final stage of the burst, the satellites congregate $\left(\left|z_{s}\right| \approx 1\right)$, but nevertheless remain remote from the host ( $D$ is large). This is a quite unusual state, which we attribute to the fact that the transversal Lyapunov exponent is smaller in absolute value close to the host, as one can see from Figure 5 . There is quite a long time interval $1300 \lesssim t \lesssim 1500$, where the satellites stay together but are detached from the host. This observation explains "lonely active units" in Figure 3.

\section{Conclusion}

In this paper we considered a special class of networks - social-type networks STNs. From the mathematical viewpoint, they are skew systems: one active network with interconnections, which drives another, passive network. Moreover, we assume that there no interconnections in the passive subnetwork, so that it consists of individual driven elements. Furthermore, it is natural to assume that the number of active elements is small, and the number of passive units is large. This configuration mimics what really is observed in the social networks like the facebook $[5,19]$. We, however, consider the effects related to STN for oscillatory systems. We have considered both active and passive oscillators forming a symmetric ring, with long-range interactions. Active oscillators form a chimera pattern, with a synchronous and an asynchronous domains on a ring. Our main focus was on the dynamics of passive units. We have demonstrated that they are rather correlated, what is explained by negative transversal Lyapunov exponents. A remarkable intermittent dynamics is demonstrated by passive units (satellites) which are very close to an active host. Most of the time the satellites follow the host, but there are bursts where they detach and leave the host to move for certain time alone; after that the satellites again attach to the host. Probably, such a behavior by followers could be observed in social networks as well.

We stress here that essential for our analysis was a rather small number of active oscillators. The role of this number is twofold: first, it leads to fluctuations of the force driving passive elements, and second, it leads to weak turbulence of the active oscillators which restores ergodicity in the system. Let us briefly discuss, how the effects change for large active population sizes $N$. In this case chimera will move so slowly that the time where ergodicity establishes is not available. Thus, one should distinguish passive oscillators in the synchronous and the asynchronous domains. Even larger effect on the dynamics of passive elements is due to smallness of finite-size fluctuations. Indeed, in the thermodynamic limit $N \rightarrow \infty$ the field acting on oscillators is stationary in the proper rotating reference frame. Thus, passive elements will have negative LEs in the synchronous domain, and vanishing LEs in the asynchronous domain. The correlations, which are due to negative LEs, disappear in this limit, and can be expected to be very weak for large population sizes $N$.

\section{ApPendix A}

Here we discuss phase slips in the profile of passive oscillators. Due to irregular driving, even in the asynchronous domain there is a stable (time-varying) phase to which neighboring passive oscillators converge with the rate given by the average negative Lyapunov exponent $\lambda_{t}$. Because the phases live on a circle, the stable phase and the stable phase shifted by $2 \pi$ are equivalent, and both are attracting. This means that between them should be an unstable phase, to which corresponds a positive Lyapunov exponent $\lambda_{+}$. A phase slip is a continuous spatial profile $\vartheta(x, t)$ of the phase of passive oscillators, tending to the stable phase at large negative $x$ and tending to the stable phase shifted by $\pm 2 \pi$ for large positive $x$. The evolution of this slip is as follows: the tails get attracted to the stable positions, while in the middle the profile will go through the value of the unstable 
phase. Formally speaking, in the spatially continuous case such a profile never disappears, but it becomes more and more step-like, the width of the step $\delta$ decreases in time exponentially as $\delta(t)=\delta(0) \exp \left[-\lambda_{+} t\right]$. If we have a finite number of equidistributed passive oscillators $M$, then the distance between them is $\sim M^{-1}$. The slip will be not observed in this setup, if there is no any point at the slip width $\delta<M^{-1}$, what gives an estimate for the life time of the slip $T_{\text {slip }} \sim \frac{\ln M}{\lambda_{+}}$. One can see that slips disappear quite fast even for very large numbers of passive oscillators $M$.

Acknowledgements. The work was supported by the Russian Science Foundation (grant Nr. 17-12-01534) and by DFG (grant PI 220/22-1).

\section{REFERENCES}

[1] D.M. Abrams and S.H. Strogatz, Chimera states for coupled oscillators. Phys. Rev. Lett. 93 (2004) 174102.

[2] M. Bolotov, L. Smirnov, G. Osipov and A. Pikovsky, Simple and complex chimera states in a nonlinearly coupled oscillatory medium. Chaos 28 (2018) 045101.

[3] G. Bordyugov, A. Pikovsky, and M. Rosenblum, Self-emerging and turbulent chimeras in oscillator chains. Phys. Rev. E 82 (2010) 035205.

[4] G.L. Eyink and K.R. Sreenivasan, Onsager and the theory of hydrodynamic turbulence. Rev. Mod. Phys. 78 (2006) 87-135.

[5] J. Gerson, A.C. Plagnol and P.J. Corr, Passive and Active Facebook Use Measure (PAUM): Validation and relationship to the Reinforcement Sensitivity Theory. Person. Individ. Differ. 117 (2017) 81-90.

[6] R.J. Goldschmidt, A. Pikovsky and A. Politi, Blinking chimeras in globally coupled rotators. Chaos 29 (2019) 071101.

[7] Y. Kuramoto and D. Battogtokh, Coexistence of coherence and incoherence in nonlocally coupled phase oscillators. Nonlinear Phenom. Complex Syst. 5 (2002) 380-385.

[8] C.R. Laing, The dynamics of chimera states in heterogeneous Kuramoto networks. Physica D 238 (2009) $1569-1588$.

[9] Y. Maistrenko, O. Sudakov, O. Osiv and V. Maistrenko, Chimera states in three dimensions. N. J. Phys. 17 (2015) 073037.

[10] Y.L. Maistrenko, A. Vasylenko, O. Sudakov, R. Levchenko and V.L. Maistrenko, Cascades of multiheaded chimera states for coupled phase oscillators. Int. J. Bifurc. Chaos 24 (2014) 1440014.

[11] O.E. Omel'chenko, Coherence-incoherence patterns in a ring of non-locally coupled phase oscillators. Nonlinearity 26 (2013) 2469.

[12] O.E. Omel'chenko and E. Knobloch, Chimerapedia: coherence-incoherence patterns in one, two and three dimensions. $N$. $J$. Phys. 21 (2019) 093034.

[13] M.J. Panaggio and D.M. Abrams, Chimera states: coexistence of coherence and incoherence in networks of coupled oscillators. Nonlinearity 28 (2015) R67-R87.

[14] F. Peter, C.C. Gong and A. Pikovsky, Microscopic correlations in the finite-size Kuramoto model of coupled oscillators. Phys. Rev. E 100 (2019) 032210.

[15] A. Pikovsky, M. Rosenblum and J. Kurths, Synchronization. A Universal Concept in Nonlinear Sciences. Cambridge University Press, Cambridge (2001).

[16] L. Schmidt and K. Krischer, Clustering as a prerequisite for chimera states in globally coupled systems. Phys. Rev. Lett. 114 (2015) 034101.

[17] L. Schmidt, K. Schönleber, K. Krischer and V. Garc'ia-Morales, Coexistence of synchrony and incoherence in oscillatory media under nonlinear global coupling. Chaos 24 (2014).

[18] L. Smirnov, G. Osipov and A. Pikovsky, Chimera patterns in the Kuramoto-Battogtokh model. J. Phys. A: Math. Theor. 50 (2017) 08LT01.

[19] B.M. Trifiro and J. Gerson, Social media usage patterns: Research note regarding the lack of universal validated measures for active and passive use. SocialMedia + Society (2019) 1-4.

[20] M. Wolfrum and O.E. Omel'chenko, Chimera states are chaotic transients. Phys. Rev. E 84 (2011) 015201.

[21] J. Xie, E. Knobloch and H.-C. Kao, Multicluster and traveling chimera states in nonlocal phase-coupled oscillators. Phys. Rev. E 90 (2014) 022919.

[22] A. Yeldesbay, A. Pikovsky and M. Rosenblum, Chimeralike states in an ensemble of globally coupled oscillators. Phys. Rev. Lett. 112 (2014) 144103.

[23] M. Zaks and A. Pikovsky, Chimeras and complex cluster states in arrays of spin-torque oscillators. Sci. Rep. 7 (2017) 4648. 